\title{
Identification and resolution of drug-related problems in a tertiary hospital respiratory unit in China
}

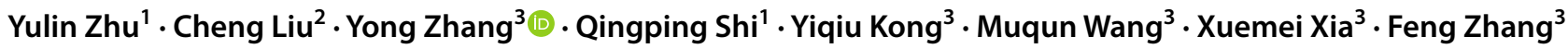

Received: 24 December 2018 / Accepted: 9 October 2019 / Published online: 25 October 2019

(c) The Author(s) 2019

\begin{abstract}
Background The prevalence of drug-related problems in patients hospitalized at respiratory care units is unknown in mainland China. Objective To identify and categorize drug-related problems in a respiratory care unit in China. Setting Respiratory care unit in a tertiary university hospital in China. Methods Clinical pharmacy services were introduced and documented during an 18-months study period. The problems were categorized using the Pharmaceutical Care Network Europe DRP classification tool V8.02. Main outcome measures Problems and causes of drug-related problems, interventions proposed, and outcome of pharmacy recommendations. Results A total of 474 patients were reviewed, 164 patients had DRPs (34.6\%). Total 410 problems were identified, an average of 2.5 per patient. Treatment effectiveness was the major type of problem detected $(219 ; 53.4 \%)$ followed by treatment safety $(140 ; 34.1 \%)$. The most common causes of the problems were patientrelated (25.8\%), drug selection (24.0\%), and drug use process (23.4\%). Pharmacist made 773 interventions; average 1.9 per drug-related problem. A total of $96.2 \%$ of these interventions were accepted leading to solving $81.9 \%$ of the identified problems. Conclusion There is a high prevalence of drug-related problems in patients hospitalized at the respiratory unit of this clinic. Clinical pharmacists should focus on improving prescribing practice and patient counseling.
\end{abstract}

Keywords China $\cdot$ Clinical pharmacy services $\cdot$ Drug-related problems $\cdot$ PCNE $\cdot$ Pharmaceutical care $\cdot$ Respiratory care

\section{Impact on Practice}

- Clinical pharmacy services in a respiratory care unit contributes to the identification and resolution of drugrelated problems.

- Clinical pharmacists should focus on improving prescribing practice and patient counseling.

Yong Zhang

zsuzy@126.com

1 Department of Pharmacy, The First Affiliated Hospital of Bengbu Medical College, Bengbu, Anhui, China

2 Critical Care Medicine, The First Affiliated Hospital of Bengbu Medical College, Bengbu, Anhui, China

3 Pulmonary and Critical Care Medicine, The First Affiliated Hospital of Bengbu Medical College, Bengbu, Anhui, China

\section{Introduction}

Respiratory diseases are one of the most common diseases in the world [1] and caused 3.8 million deaths in 2015 worldwide [2]. The report released by the Forum of International Respiratory Societies identifies five conditions that primarily contribute to the global burden of respiratory disease: asthma, chronic obstructive pulmonary disease (COPD), acute respiratory infections, tuberculosis, and lung cancer [3]. Respiratory diseases were the third most common cause of death in China in 2015 [4].

Clinical pharmacy services can contribute to reducing hospital mortality rates [5]. Several studies have shown that pharmacist participation in respiratory disease management has an impact on patient care: they can educate patients on diseases and medications; improve medication compliance, disease control, and therapy outcomes; and reduce hospital admissions and healthcare-related costs [6-9]. Identifying and resolving drug-related problems (DRPs) associated with prescription orders are the core activities of pharmaceutical care. A DRP is "an event or circumstance involving drug therapy that actually or potentially interferes with desired 
health outcomes" [10]. DRPs may lead to increased morbidity, mortality, and healthcare costs [11].

Most research in respiratory disease management has been conducted in Western countries, and clinical pharmacy service in this patient care field is still in development in China. The extent and characteristics of DRPs in respiratory medicine units are unknown in mainland China, and the impact of pharmacist participation in patient care needs further research. We introduced a respiratory care pharmacy service at a Chinese hospital in 2015. This paper is a description of our findings.

\section{Aim of the study}

The objective was to categorize DRPs identified in a respiratory care unit and to assess interventions provided by pharmacists.

\section{Ethics approval}

The study was approved by the First Affiliated Hospital of Bengbu Medical College Institutional Review Board (No. 2015092).

\section{Methods}

\section{Setting}

The study was consducted in the respiratory medicine department from January 1, 2015, and June 30, 2016, at the First Affiliated Hospital of Bengbu Medical College, a 1,800-bed teaching hospital servicing a population of 30 million people in northern Anhui province, East China. The respiratory medicine unit has 120 beds, including 8 intensive care beds. Patients were cared for by a multi-disciplinary team (MDT) consisted of physicians, nurses, and one clinical pharmacist. The clinical pharmacist had completed residency training with a sub-specialty in infectious diseases accredited by the China Ministry of Health and had 5 years of hospital practice experience. The responsibilities of the clinical pharmacist were: (1) participating in daily MDT round and case discussions, (2) providing drug information and therapy consultations to the MDT, (3) reviewing medication orders to identify DRPs and proposing clinical interventions to resolve the DRPs identified. The hospital has computerized physician order entry (CPOE) with clinical decision support for physician prescribing and pharmacist order verification.

\section{Study design and data collection}

The pharmacist reviewed medication orders for all patients admitted to the respiratory medicine unit within the study period. The following patient demographic and clinical information were collected: gender, age, length of hospital stay, smoking and drinking habits, diagnosis on admission, and concomitant chronic diseases.

The pharmacist reconciled patients' current medication lists, and an advanced medication review was performed for each patient by the clinical pharmacist within $24-48 \mathrm{~h}$ of patient admission to the unit during weekdays based on data collected from patient's medical records, a direct patientpharmacist interview and rounding with the MDT. During MDT rounds, pharmacists also paid attention to how nurses administered drugs when possible. The clinical pharmacist used relevant treatment guidelines from major Chinese and international associations to detect DRPs, and standardized databases such as the China National Formulary, Medscape, and UpToDate, to assist with the calculation of appropriate doses based on creatinine clearance (Cockcroft-Gault equation). Major treatment guidelines referenced were from the Infectious Diseases Society of America (IDSA) [12], the Chinese Thoracic Society [13], The Global Initiative for Asthma (GINA) [14] and The Global Initiative for Chronic Obstructive Lung Disease (GOLD) [15]). The Chinese guidelines are similar to international guidelines, except drugs are tailored based on Chinese formulary. DRPs identified were verified with the MDT, and appropriate pharmacy interventions to resolve the identified DRPs were discussed with the MDT. The clinical pharmacist evaluated the outcome of each recommendation, and the physicians confirmed the results.

\section{Data and statistical analysis}

The identified DRPs, causes, interventions, and outcomes were characterized using the Pharmaceutical Care Network Europe (PCNE) DRP classification V8.02, which was last updated in 2017 [16]. The rate of DRPs was estimated by calculating the number of DRPs per patient. One Problem (P) may have multiple Causes (C), and lead to more than one Intervention (I), but it leads to only one Outcome (O). A descriptive analysis was conducted on the patient's demographics, clinical characteristics, causes of DRPs, and types and outcomes of interventions. Frequencies and percentages were calculated for categorical variables. All analyses were performed using SPSS 18.0. Data were presented as medians and interquartile ranges (IQRs) (25-75th percentiles). $T$ test, variance analysis, $\chi^{2}$ analysis, and rank-sum test were used in the analysis. $p$ values $<0.05$ were considered statistically significant. 


\section{Results}

\section{Patient characteristics}

A total of 474 patients was hospitalized during the 18 -month study period, and $164(34.6 \%)$ patients had DRPs requiring pharmacist interventions. Table 1 shows the demographic and clinical characteristics of these patients: the average age was 65.0 years, and $58.9 \%$ were males. The average length of hospital stay was 13.0 days, and patients with DRPs had a longer duration of hospital stay, 15.0 days versus 12.5 days. The top three reasons for admission were pneumonia (31.9\%), lung cancer $(25.7 \%)$, and acute exacerbation of COPD/Cor Pulmonale (23.8\%). Total $33.8 \%$ of patients had concomitant cardiovascular diseases followed by diabetes $(17.7 \%)$ and cerebrovascular diseases (12.4\%). More DRPs were identified in patients with cardiovascular diseases and diabetes. The top three medication classes taken were: anti-infective

Table 1 Patient demographic and clinical characteristics

\begin{tabular}{|c|c|c|c|}
\hline Number & Total 474(100\%) & With DRP 164(34.6\%) & Without DRP 310(65.4\%) \\
\hline \multicolumn{4}{|l|}{ Characteristics } \\
\hline Sex, male & $279(58.9)$ & $104(63.4)$ & $175(56.5)$ \\
\hline Age, years & $65.0 \pm 14.5$ & $65.5 \pm 14.8$ & $64.0 \pm 14.1^{*}$ \\
\hline Length of hospital stay, days & $13.0 \pm 13.3$ & $15.0 \pm 14.6$ & $12.5 \pm 11.8 * * *$ \\
\hline History of smoking habits & $118(24.9)$ & $43(26.2)$ & $75(24.2)$ \\
\hline History of drinking habits & $68(14.3)$ & $23(14.0)$ & $45(14.5)$ \\
\hline \multicolumn{4}{|l|}{ Admission diagnosis } \\
\hline Pneumonia & $151(31.9)$ & $50(30.5)$ & 101(32.6) \\
\hline $\mathrm{AECOPD}^{\mathrm{a}} / \mathrm{COPD}^{\mathrm{b}}$ & $113(23.8)$ & $42(25.6)$ & $71(22.9)$ \\
\hline Lung cancer & $122(25.7)$ & $42(25.6)$ & $80(25.8)$ \\
\hline Pulmonary fibrosis & $28(5.9)$ & $10(6.1)$ & $18(5.8)$ \\
\hline Bronchiectasis & $26(5.5)$ & $10(6.1)$ & $16(5.2)$ \\
\hline Thrombus & $21(4.4)$ & $7(4.3)$ & $14(4.5)$ \\
\hline Asthma & $13(2.7)$ & $3(1.8)$ & $10(3.2)$ \\
\hline \multicolumn{4}{|l|}{ Concomitant diseases } \\
\hline Cardiovascular diseases & $160(33.8)$ & $74(45.1)$ & $86(27.7)^{* * *}$ \\
\hline Diabetes & $84(17.7)$ & $43(26.2)$ & $41(13.2)^{* *}$ \\
\hline Cerebrovascular disease & $59(12.4)$ & $23(14.0)$ & $36(11.6)$ \\
\hline Chronic kidney disease & $23(4.9)$ & $11(6.7)$ & $12(3.9)$ \\
\hline Autoimmune disease & $20(4.2)$ & $10(6.1)$ & $10(3.2)$ \\
\hline Chronic liver disease & $13(2.7)$ & $7(4.3)$ & $6(1.9)$ \\
\hline Epilepsy & $10(2.1)$ & $4(2.4)$ & $6(1.9)$ \\
\hline \multicolumn{4}{|l|}{ Medication } \\
\hline Cardiovascular drugs & $184(38.8)$ & $91(55.5)$ & $93(30.0) * * *$ \\
\hline Anti-infective drugs & $369(77.8)$ & $135(79.3)$ & $234(75.5)$ \\
\hline Nutrition/electrolyte/vitamin & $135(28.5)$ & $52(31.7)$ & $83(26.8)$ \\
\hline Respiratory drugs & $188(39.7)$ & $66(40.2)$ & $122(39.4)$ \\
\hline Endocrine system drugs & $100(47.4)$ & $49(29.9)$ & $51(16.5)^{* *}$ \\
\hline Digestive system drugs & $139(29.3)$ & $45(27.4)$ & $94(30.3)$ \\
\hline Traditional Chinese Medicine drugs & $53(11.2)$ & $20(12.2)$ & $33(10.6)$ \\
\hline Nervous system drugs & $47(9.9)$ & $18(11.0)$ & $29(9.4)$ \\
\hline Liver protective drugs & $17(3.6)$ & $10(6.1)$ & $7(2.3)$ \\
\hline Glucocorticoid & $132(27.8)$ & $42(25.6)$ & $90(29.0)$ \\
\hline Lung cancer chemotherapy drugs/analgesics & $122(25.7)$ & $42(25.6)$ & $80(25.8)$ \\
\hline Mean number of drugs taken & & $13.2 \pm 5.2$ & $11.2 \pm 4.9 * * *$ \\
\hline
\end{tabular}

${ }^{a}$ Acute exacerbation of the chronic obstructive pulmonary disease

${ }^{\mathrm{b}}$ Chronic obstructive pulmonary disease

$* p<0.05 ; * * p<0.005 ; * * * p<0.0001$ 
drugs $(77.8 \%)$ followed by drugs for the endocrine system $(47.4 \%)$, and drugs for the respiratory tract $(39.7 \%)$. Patients with DRPs exhibited greater use of cardiovascular and endocrine system drugs. Patients with DRPs took more drugs on average compared to patients without DRPs, $13.2 \pm 5.2$ versus $11.2 \pm 4.9(p<0.0001)$.

\section{Identified drug-related problems}

A total of 410 DRPs were identified, an average of 2.5 per patient (Table 2). Treatment effectiveness P1 was the major type of DRPs (219 of 410 DRPs; 53.4\%) followed by treatment safety P2 $(140 ; 34.1 \%)$. Within the treatment effectiveness P1 category, the effect of drug treatment not optimal P1.2 was the dominant category (120/219; $54.8 \%)$.

\section{Causes of drug-related problems identified}

A total of 492 DRP causes were identified (Table 3). Patient-related C7 category was the primary cause of DRPs $(127 ; 25.8 \%)$ followed by drug selection C1 (118; $24.0 \%)$ and drug use process C6 $(115 ; 23.4 \%)$. Within these top three domains, the number one subcategory for each domain was: patient uses/takes less drug than prescribed or does not take the drug at all C7.1 (35/127; $27.6 \%$ ) in the patient-related domain; inappropriate duplication of therapeutic group or active ingredient $\mathrm{C} 1.5$ $(36 / 118 ; 30.5 \%)$ in the drug selection domain, and inappropriate timing of administration and/or dosing intervals C6.1 $(84 / 115 ; 73.0 \%)$ in the drug use process domain.

\section{Proposed interventions to resolve the identified DRPs}

A total of 773 interventions were suggested; average 1.9 interventions per DRP identified (Table 4). Most interventions occurred at the drug-level I3 $(341 ; 44.1 \%)$ followed by at the prescriber-level I1 $(270 ; 34.9 \%)$. At the drug-level, dosage change I3.2 was the major sub-domain $(121 / 341 ; 35.5 \%)$; and intervention proposed to prescriber I1.3 $(174 / 270 ; 64.4 \%)$ was the major sub-category at the prescriber-level.

\section{Acceptance of the intervention proposals}

Total $744(96.2 \%)$ interventions were accepted and regarded as clinically relevant. Among these accepted interventions, $662(89.0 \%)$ were accepted and fully implemented by prescribers or patients (Table 5).

\section{Outcomes of interventions}

A total of 410 DRPs were identified, and 336 (81.9\%) DRPs were solved, while 42 (10.3\%) DRPs were partially solved, and 32 (7.8\%) DRPs were unresolved (Table 6). Among the not solved $\mathrm{O} 3$ domain, lack of cooperation from patient $\mathrm{O} 3.1$ or prescriber $\mathrm{O} 3.2$ were the major causes of failed intervention outcome.

\section{Discussion}

To the best of our knowledge, this is the first prospective study describing the prevalence of drug-related problems in patients admitted to a respiratory care unit in mainland China. DRPs are common in this patient population. This highlights the needs for clinical pharmacy service in this specialized patient care unit.

Total $34.6 \%$ of the patients in our study had DRPs, and the average number of DRPs per patient was 2.5. We could not find the prevalence of DRPs in respiratory care units in published studies for a direct comparison. Some studies showed a significantly different mean number of DRPs per patient in Chinese hospitals. For example, a study in hospitalized surgical patients in China reported a mean of

Table 2 Identified problems according to the PCNE DRP classification tool V8.02

\begin{tabular}{lll}
\hline Primary domain & Type of problem & $\begin{array}{c}\text { Total num- } \\
\text { ber }=410 \\
(100 \%)\end{array}$ \\
\hline Treatment effectiveness P1 & & $91(22.2)$ \\
& No effect of drug treatment P1.1 & $120(29.3)$ \\
& Effect of drug treatment not optimal P1.2 & $8(1.9)$ \\
Treatment Safety P2 & Untreated symptoms or indication P1.3 & $140(34.1)$ \\
Others P3 & Adverse drug event (possibly) occurring P2.1 & $12(3.0)$ \\
& Problem with cost-effectiveness of the treatment P3.1 & $39(9.5)$ \\
& Unnecessary drug-treatment P3.2 & $0(0)$ \\
\hline
\end{tabular}


Table 3 Identified causes according to the PCNE DRP classification tool V8.02

\begin{tabular}{|c|c|c|}
\hline Primary domain & Cause of the problem & $\begin{array}{l}\text { Total num- } \\
\text { ber }=492 \\
(100 \%)\end{array}$ \\
\hline \multirow[t]{7}{*}{ Drug selection $\mathrm{C} 1$} & Inappropriate drug according to guidelines/formulary $\mathrm{C} 1.1$ & $18(3.6)$ \\
\hline & Inappropriate drug (within guidelines but otherwise contra-indicated) C1.2 & $13(2.6)$ \\
\hline & No indication for drug C1.3 & $13(2.6)$ \\
\hline & Inappropriate combination of drugs or drugs and herbal medication $\mathrm{C} 1.4$ & $26(5.3)$ \\
\hline & Inappropriate duplication of therapeutic group or active ingredient $\mathrm{C} 1.5$ & $36(7.4)$ \\
\hline & No drug treatment in spite of existing indication C1.6 & $4(0.8)$ \\
\hline & Too many drugs prescribed for indication $\mathrm{C} 1.7$ & $8(1.7)$ \\
\hline Drug form $\mathrm{C} 2$ & Inappropriate drug form (for this patient) $\mathrm{C} 2.1$ & $9(1.8)$ \\
\hline \multirow[t]{5}{*}{ Dose selection C3 } & Drug dose too low $\mathrm{C} 3.1$ & $11(2.2)$ \\
\hline & Drug dose too high $\mathrm{C} 3.2$ & $40(8.1)$ \\
\hline & Dosage regimen not frequent enough $\mathrm{C} 3.3$ & $13(2.6)$ \\
\hline & Dosage regimen too frequent $\mathrm{C} 3.4$ & $20(4.1)$ \\
\hline & Dose timing instructions wrong, unclear or missing C3.5 & $11(2.3)$ \\
\hline \multirow[t]{2}{*}{ Treatment duration $\mathrm{C} 4$} & Duration of treatment too short $\mathrm{C} 4.1$ & $3(0.6)$ \\
\hline & Duration of treatment too long $\mathrm{C} 4.2$ & $7(1.4)$ \\
\hline \multirow[t]{4}{*}{ Dispensing C5 } & Prescribed drug not available C5.1 & $0(0)$ \\
\hline & Necessary information not provided C5.2 & $0(0)$ \\
\hline & Wrong drug, strength or dosage advised (OTC) C5.3 & $0(0)$ \\
\hline & Wrong drug or strength dispensed C5.4 & $0(0)$ \\
\hline \multirow[t]{6}{*}{ Drug use process C6 } & Inappropriate timing of administration and/or dosingintervals C6.1 & $84(17.1)$ \\
\hline & Drug under-administered C6.2 & $6(1.3)$ \\
\hline & Drug over-administered C6.3 & $1(0.3)$ \\
\hline & Drug not administered at all C6.4 & $15(3.1)$ \\
\hline & Wrong drug administered C6.5 & $0(0)$ \\
\hline & Drug administered via wrong route C6.6 & $9(0.8)$ \\
\hline \multirow[t]{9}{*}{ Patient related $\mathrm{C} 7$} & Patient uses/takes less drug than prescribed or does nottake the drug at all C7.1 & $35(7.1)$ \\
\hline & Patient uses/takes more drug than prescribed C7.2 & $0(0)$ \\
\hline & Patient abuses drug (unregulated overuse) C7.3 & $5(1.0)$ \\
\hline & Patient uses unnecessary drug C7.4 & $10(1.9)$ \\
\hline & Patient takes food that interacts $\mathrm{C} 7.5$ & $8(1.5)$ \\
\hline & Patient stores drug inappropriately C7.6 & $0(0)$ \\
\hline & Inappropriate timing or dosing intervals $\mathrm{C} 7.7$ & $26(5.3)$ \\
\hline & Patient administers/uses the drug in a wrong way $\mathrm{C} 7.8$ & $19(3.9)$ \\
\hline & Patient unable to use drug/form as directed C7.9 & $24(4.9)$ \\
\hline \multirow[t]{3}{*}{ Other $\mathrm{C} 8$} & No or inappropriate outcome monitoring (including TDM) C8.1 & $18(3.6)$ \\
\hline & Other cause; specify C 8.2 & $0(0)$ \\
\hline & No obvious cause $\mathrm{C} 8.3$ & $0(0)$ \\
\hline
\end{tabular}

0.3 DRPs per patient using the PCNE classification V8.02 [17]. The study in cardiovascular units in Northern Cyprus demonstrates that $61 \%$ of patients had DRPs using the PCNE classification V6.2, and the average DRPs per patient was 1.6 [18]. Another study done in an internal medicine ward in Turkey indicates that $80 \%$ of patients had DRPs using PCNE classification V7.0, an average of 1.6 DRPs per patient [19]. The methodological issues of reporting, study population and setting, and classification could contribute to the variations of DRP prevalence among studies [20].

In our analysis, there were no significant differences between males and females regarding the number of DRPs. However, there was a connection between an increased hospital stay and the development of DRPs. This association was also observed in the Turkish study in internal medicine patients [19]. More medications could be prescribed 
Table 4 Proposed interventions according to the PCNE DRP classification tool V8.02

\begin{tabular}{lll}
\hline Primary domain & Type of intervention & $\begin{array}{l}\text { Total num- } \\
\text { ber }=773 \\
(100 \%)\end{array}$ \\
\hline No intervention I0 & & $0(0)$ \\
At prescriber level I1 & No Intervention I0.1 & $67(8.7)$ \\
& Prescriber informed only I1.1 & $13(1.7)$ \\
& Prescriber asked for information I1.2 & $174(22.5)$ \\
At patient level I2 & Intervention proposed to prescriber I1.3 & $16(2.1)$ \\
& Intervention discussed with prescriber I1.4 & $52(6.7)$ \\
& Patient (drug) counseling I2.1 & $12(1.6)$ \\
& Written information provided (only) I2.2 & $2(0.3)$ \\
At drug level I3 & Patient referred to prescriber I2.3 & $87(11.3)$ \\
& Spoken to family member/caregiver I2.4 & $66(8.5)$ \\
& Drug changed to .... I3.1 & $121(15.7)$ \\
& Dosage changed to .... I3.2 & $15(1.9)$ \\
& Formulation changed to .... I3.3 & $7(0.9)$ \\
& Instructions for use changed to .... I3.4 & $102(13.2)$ \\
& Drug stopped I3.5 & $30(3.9)$
\end{tabular}

Table 5 Acceptance of the intervention proposals according to the PCNE DRP classification tool V8.02

\begin{tabular}{|c|c|c|}
\hline Primary domain & Type of implementation & $\begin{array}{l}\text { Total num- } \\
\text { ber }=773 \\
(100 \%)\end{array}$ \\
\hline \multirow[t]{4}{*}{ Intervention accepted (by prescriber or patient) A1 } & Intervention accepted and fully implemented A1.1 & $662(85.6)$ \\
\hline & Intervention accepted, partially implemented A1.2 & $55(7.1)$ \\
\hline & Intervention accepted but not implemented A1.3 & $10(1.3)$ \\
\hline & Intervention accepted, implementation unknown A1.4 & $17(2.2)$ \\
\hline \multirow[t]{4}{*}{ Intervention not accepted (by prescriber or patient) A2 } & Intervention not accepted: not feasible A2.1 & $3(0.4)$ \\
\hline & Intervention not accepted: no agreement A2.2 & $13(1.7)$ \\
\hline & Intervention not accepted: other reason (specify) A2.3 & $0(0)$ \\
\hline & Intervention not accepted: unknown reason A2.4 & $4(0.5)$ \\
\hline \multirow[t]{2}{*}{ Other(no information on acceptance) A3 } & Intervention proposed, acceptance unknown A3.1 & $9(1.2)$ \\
\hline & Intervention not proposed A3.2 & $0(0)$ \\
\hline
\end{tabular}

Table 6 Outcomes according to the PCNE DRP classification tool V8.02

\begin{tabular}{lll}
\hline Primary domain & Outcome of intervention & $\begin{array}{l}\text { Total num- } \\
\text { ber }=410 \\
(100 \%)\end{array}$ \\
\hline Not known O0 & Problem status unknown O0.1 & 0 \\
Solved O1 & Problem totally solved O1.1 & $336(81.9)$ \\
Partially solved O2 & Problem partially solved O2.1 & $42(10.3)$ \\
Not solved O3 & Problem not solved, lack of cooperation of patient O3.1 & $16(4.0)$ \\
& Problem not solved, lack of cooperation of prescriber O3.2 & $13(3.1)$ \\
& Problem not solved, intervention not effective O3.3 & $3(0.6)$ \\
& No need or possibility to solve problem O3.4 & 0 \\
\hline
\end{tabular}


to patients with longer hospital stays, and this might have contributed to an increased number of DRPs.

The causes of the DRPs identified were mainly at the patient level, drug selection, and drug use process, and these accounted for $73.5 \%$ of the causes. This indicates that a patient's drug use behavior, physician's prescription behavior, and nurse drug administration all could affect the appropriate use of drugs. Patients were allowed to keep and take their own medications before and during admission, which could be the cause for DRPs. This demonstrates the necessity of medication reconciliation by pharmacists. One patient-level example was: a patient who needed government assistance support had multiple severe COPD exacerbations requiring hospitalizations. Upon the clinical pharmacist interview to the patient, we found that this patient had insufficient knowledge about his medications and his poor inhaler use skills led to his poor COPD control. Studies have demonstrated that treatment-to-goal education with both verbal instructions and device demonstration is an essential first step toward improving self-management and health outcomes for hospitalized patients with COPD, especially among patients with lower levels of health literacy $[21,22]$. One example at the prescriber drug selection level was cefuroxime combined with moxifloxacin was frequently prescribed for admitted community-acquired pneumonia patients regardless of the patient's exposure to the environment, medication history, and other characteristics. One example at the nurse drug administration level was antibacterial drug dosing interval such as $\mathrm{q} 6 \mathrm{~h}, \mathrm{q} 8 \mathrm{~h}$, or q12 $\mathrm{h}$ were often administrated at wrong time intervals.

Compared to patients without DRPs, patients with DRPs took more drugs, especially cardiovascular and endocrine system drugs. The three primary medication classes associated with DRPs were anti-infective drugs (79.3\%), cardiovascular drugs (55.5\%), and respiratory drugs (40.2\%). The drug types were consistent with the admission diagnoses such as pneumonia (30.5\%), AECOPD/COPD (25.6\%), and lung cancer (25.6\%). However, the cardiovascular system drugs ranked second, while lung cancer chemotherapy drugs/analgesics and glucocorticoids ranked seventh. In our patient population, cardiovascular disease was the most common comorbidity (45.1\%), leading to increased DRPs. In contrast, chemotherapy for lung cancer was generally followed by relevant guidelines with a few occurrences of DRPs. These findings suggest that when providing clinical services at respiratory units, pharmacists should focus on patients with comorbidities taking drugs other than respiratory drugs. Specialists may, in general, have less knowledge about these drugs and disease conditions. The added value of clinical pharmacists is mainly on DRPs in general, and the overview of complete pharmacotherapy.
The safety of drug treatment accounted for almost $40 \%$ of the types of DRPs identified in our study. Some of the examples of DRPs identified were: a patient with pulmonary infection was treated with cefoperazone/sulbactam for 14 days with an undetected prothrombin time of $64 \mathrm{~s}$; voriconazole was dosed without a dosing adjustment for an invasive pulmonary fungal infection in a patient with liver function of the Child-Pugh grade C; and a 65-yearold male epilepsy patient took carbamazepine for 15 years with a development of aplastic anemia. The specific role of the clinical pharmacist in improving medication safety is increasingly important, especially for patients admitted to an intensive care unit due to high risk for prescribing errors and related adverse drug events [23, 24]. Pharmacists should actively monitor patients' drug therapy with consideration of relevant laboratory parameters. Recommendations on optimizing the clinical decision support in the CPOE system should also be proposed at the hospital level.

In our study, treatment effectiveness was the dominant type of DRPs, and this was similar to the study evaluating clinical pharmacy services to cardiovascular clinics in Northern Cyprus [18]. Patient-related category and drug selection accounted for approximately 50\% causes of the DRPs in that study. In the Turkish study, drug selection was the primary cause of DRPs. Pharmacists made an average 1.9 interventions for each DRP identified in our study, and the acceptance of pharmacists' interventions was high, $96.2 \%$, which is comparable to the findings in the Northern Cyprus study (93.1\%). In our study, intervention accepted and fully implemented was $85.6 \%$; this was similar to the Swiss University Hospital study of $83.0 \%$ [25] but lower than the study in Italy [26]. This high rate of acceptance demonstrates that pharmacist's interventions were relevant and highly useful for physicians, especially in determining the optimal drug dosage for patients. The high acceptance and implementation of pharmacist's interventions led to more than $90 \%$ of the DRPs identified solved or partially solved.

Our study has the following limitations: (1) it was conducted in a single hospital, and the DRP patterns may not be generalizable for other departments or other hospitals in China, (2) the small size of the study group, which only included 164 patients and (3) medication review was performed by one pharmacist and no validation of this review or study data was performed by another pharmacist. Furthermore, our study only assessed pharmacists' impact on identifying and resolving DRPs related to patients' pharmacotherapy, the relationship between the outcome of DRPs and patients' clinical outcome requires future research. 


\section{Conclusion}

A high rate of DRPs is observed in patients hospitalized in a respiratory care unit in China. A total $34.6 \%$ of the patients in our study had drug-related problems, and the average number of DRPs per patient was 2.5. The study also demonstrates that clinical pharmacists can identify DRPs and make clinical interventions to resolve the identified DRPs.

Funding This work was supported by the Key Research and Development Program of Anhui Province (Grant No. 1804h08020287).

Conflicts of interest All authors declare that they have no conflict of interest.

Open Access This article is distributed under the terms of the Creative Commons Attribution 4.0 International License (http://creativeco mmons.org/licenses/by/4.0/), which permits unrestricted use, distribution, and reproduction in any medium, provided you give appropriate credit to the original author(s) and the source, provide a link to the Creative Commons license, and indicate if changes were made.

\section{References}

1. GBD 2013 DALYs and HALE Collaborators, Murray Christopher JL, Barber Ryan M, et al. Global, regional, and national disability-adjusted life years (DALYs) for 306 diseases and injuries and healthy life expectancy (HALE) for 188 countries, 1990-2013: quantifying the epidemiological transition. Lancet. 2015;386(10009):2145-91.

2. GBD 2015 Mortality and Causes of Death Collaborators. Global, regional, and national life expectancy, all-cause mortality, and cause-specific mortality for 249 causes of death, 1980-2015: a systematic analysis for the Global Burden of Disease Study 2015. Lancet. 2016;386(10053):1459-544.

3. Ferkol T, Schraufnagel D. The global burden of respiratory disease. Ann Am Thorac Soc. 2014;11(3):404-6.

4. Sun W, Zhou Y, Zhang Z, Cao L, Chen W. The trends in cardiovascular diseases and respiratory diseases mortality in Urban and Rural China, 1990-2015. Int J Environ Res Public Health. 2017;14(11):1391.

5. Bond CA, Raehl CL, Franke T. Clinical pharmacy services and hospital mortality rates. Pharmacotherapy. 1999;19(5):556-64.

6. Cawley MJ, Warning WJ 2nd. Impact of a pharmacist-driven spirometry clinic service within a community family health center: a 5-year retrospective review. J Res Pharm Pract. 2018;7(2):88-94.

7. Ottenbros S, Teichert M, de Groot R, Griens F, Sodihardjo F, Wensing $\mathrm{M}$, et al. Pharmacist-led intervention study to improve drug therapy in asthma and COPD patients. Int J Clin Pharm. 2014;36(2):336-44.

8. Apikoglu-Rabus S, Yesilyaprak G, Izzettin FV. Drug-related problems and pharmacist interventions in a cohort of patients with asthma and chronic obstructive pulmonary disease. Respir Med. 2016;120:109-15.

9. Detoni KB, Oliveira IV, Nascimento MM, Caux TR, Alves MR, Ramalho-de-Oliveira D. Impact of a medication therapy management service on the clinical status of patients with chronic obstructive pulmonary disease. Int J Clin Pharm. 2017;39(1):95-103.

10. https://www.pcne.org/upload/files/230_PCNE_classification_ V8-02. Accessed 18 Nov 2018.

11. Kaufmann CP, Stämpfli D, Hersberger KE, Lampert ML. Determination of risk factors for drug-related problems: a multidisciplinary triangulation process. BMJ Open. 2015;20(5(3)):e006376.

12. Mandell LA, Wunderink RG, Anzueto A, Bartlett JG, Campbell GD, Dean NC, et al. Infectious diseases society of America/ American thoracic society consensus guidelines on the management of community-acquired pneumonia in adults. Clin Infect Dis. 2007;44(2):S27-72.

13. Chinese Thoracic Society. http://www.csrd.org.cn/cn/ppt_list. asp?id=1. Accessed 05 Mar 2016.

14. Global initiative for asthma(GINA). Global strategy for asthma management and prevention. https://ginasthma.org Accessed 06 Mar 2016.

15. Global Initiative for Chronic Obstructive Lung Disease (GOLD). Global strategy for the diagnosis, management and preventionof chronic obstructive pulmonary disease. https://goldcopd.org. Accessed 05 Mar 2016.

16. https://www.pcne.org/news/3/new-version-of-the-pcne-drp-class ification. Accessed 18 Nov 2018.

17. Qu C, Meng L, Wang N, Chen Y, Yang X, Wang J, et al. Identify and categorize drug-related problems in hospitalized surgical patients in China. Int J Clin Pharm. 2019;41(1):13-7.

18. Al-Baghdadi H, Koca Al-Baghdadi Ç, Abdi A, Gültekin O, Meštrović A, Demirdamar R, et al. Introducing clinical pharmacy services to cardiovascular clinics at a university hospital in Northern Cyprus. Int J Clin Pharm. 2017;39(6):1185-93.

19. Abunahlah N, Elawaisi A, Velibeyoglu FM, Sancar M. Drug related problems identified by clinical pharmacist at the Internal Medicine Ward in Turkey. Int J Clin Pharm. 2018;40(2):360-7.

20. Basger BJ, Moles RJ, Chen TF. Application of drug-related problem (DRP) classification systems: a review of the literature. Eur J Clin Pharmacol. 2014;70(7):799-815.

21. Press VG, Arora VM, Trela KC, Adhikari R, Zadravecz FJ, Liao $\mathrm{C}$, et al. Effectiveness of interventions to teach metered-dose and Diskus inhaler techniques: a randomized trial. Ann Am Thorac Soc. 2016;13(6):816-24.

22. Shah RF, Gupta RM. Video instruction is more effective than written instruction in improving inhaler technique. Pulm Pharmacol Ther. 2017;46:16-9.

23. Guchelaar HJ, Colen HB, Kalmeijer MD, Hudson PT, TeepeTwiss IM. Medication errors: hospital pharmacist perspective. Drugs. 2005;65(13):1735-46.

24. Klopotowska JE, Kuiper R, van Kan HJ, de Pont AC, Dijkgraaf MG, Lie-A-Huen L, et al. On-ward participation of a hospital pharmacist in a Dutch intensive care unit reduces prescribing errors and related patient harm: an intervention study. Crit Care. 2010;14(5):R174.

25. Lampert ML, Kraehenbuehl S, Hug BL. Drug-related problems: evaluation of a classification system in the daily practice of a Swiss University Hospital. Pharm World Sci. 2008;30(6):768-76.

26. Lombardi N, Wei L, Ghaleb M, Pasut E, Leschiutta S, Rossi P, et al. Evaluation of the implementation of a clinical pharmacy service on an acute internal medicine ward in Italy. BMC Health Serv Res. 2018;18(1):259.

Publisher's Note Springer Nature remains neutral with regard to jurisdictional claims in published maps and institutional affiliations. 